\title{
Model-Based Estimation of Iohexol Plasma Clearance for Pragmatic Renal Function Determination in the Renal Transplantation Setting
}

\author{
Tom C. Zwart ${ }^{1}$ (D) Aiko P. J. de Vries ${ }^{2,3}$ - Aline G. J. Engbers ${ }^{4} \cdot$ Ruth E. Dam ${ }^{2,3} \cdot$ Paul J. M. van der Boog ${ }^{2,3}$. \\ Jesse J. Swen ${ }^{1,5} \cdot$ Ron J. Keizer $^{6} \cdot$ R. Neil Dalton ${ }^{7}$ Henk-Jan Guchelaar ${ }^{1,5} \cdot$ Johan W. de Fijter ${ }^{2,3}$ • Dirk Jan A. R. Moes ${ }^{1,5}$
}

Accepted: 3 February 2021 / Published online: 17 April 2021

(c) The Author(s) 2021

\begin{abstract}
Background Iohexol plasma clearance-based glomerular filtration rate (GFR) determination provides an accurate method for renal function evaluation. This technique is increasingly advocated for clinical situations that dictate highly accurate renal function assessment, as an alternative to conventional serum creatinine-based methods with limited accuracy or poor feasibility. In the renal transplantation setting, this particularly applies to living renal transplant donor eligibility screening, renal transplant function monitoring and research purposes. The dependency of current iohexol GFR estimation techniques on extensive sampling, however, has limited its clinical application. We developed a population pharmacokinetic model and limited sampling schedules, implemented in the online InsightRX precision dosing platform, to facilitate pragmatic iohexol GFR assessment.

Methods Iohexol concentrations $(n=587)$ drawn $5 \mathrm{~min}$ to $4 \mathrm{~h}$ after administration were available from 67 renal transplant recipients and 41 living renal transplant donor candidates with measured iohexol GFRs of $27-117 \mathrm{~mL} / \mathrm{min} / 1.73 \mathrm{~m}^{2}$. These were split into a model development $(n=72)$ cohort and an internal validation $(n=36)$ cohort. External validation was performed with 1040 iohexol concentrations from 268 renal transplant recipients drawn between $5 \mathrm{~min}$ and $4 \mathrm{~h}$ after administration, and extended iohexol curves up to $24 \mathrm{~h}$ from 11 random patients with impaired renal function. Limited sampling schedules based on one to four blood draws within $4 \mathrm{~h}$ after iohexol administration were evaluated in terms of bias and imprecision, using the mean relative prediction error and mean absolute relative prediction error. The total deviation index and percentage of limited sampling schedule-based GFR predictions within $\pm 10 \%$ of those of the full model $\left(P_{10}\right)$ were assessed to aid interpretation.

Results Iohexol pharmacokinetics was best described with a two-compartmental first-order elimination model, allometrically scaled to fat-free mass, with patient type as a covariate on clearance and the central distribution volume. Model validity was confirmed during the internal and external validation. Various limited sampling schedules based on three to four blood draws within $4 \mathrm{~h}$ showed excellent predictive performance (mean relative prediction error $< \pm 0.5 \%$, mean absolute relative prediction error $<3.5 \%$, total deviation index $<5.5 \%, P_{10}>97 \%$ ). The best limited sampling schedules based on three to four blood draws within $3 \mathrm{~h}$ showed reduced predictive performance (mean relative prediction error $< \pm 0.75 \%$, mean absolute relative prediction error $<5.5 \%$, total deviation index $<9.5 \%, P_{10} \geq 85 \%$ ), but may be considered for their enhanced clinical feasibility when deemed justified.

Conclusions Our online pharmacometric tool provides an accurate, pragmatic, and ready-to-use technique for measured GFR-based renal function evaluation for clinical situations where conventional methods lack accuracy or show limited feasibility. Additional adaptation and validation of our model and limited sampling schedules for renal transplant recipients with GFRs below $30 \mathrm{~mL} / \mathrm{min}$ is warranted before considering this technique in these patients.
\end{abstract}

Extended author information available on the last page of the article 


\section{Key Points}

Unequivocal renal function determination is pivotal for living donor eligibility screening, transplant function monitoring and research within the renal transplantation setting. Unfortunately, current renal function evaluation methods show limited accuracy or poor clinical feasibility. Glomerular filtration rate assessment by determination of the plasma clearance of intravenous iohexol has been advocated to fill this gap. Optimisation of this technique, however, is warranted to aid its faltering clinical application.

This study describes the development of a population pharmacokinetic model and limited sampling schedules for iohexol, aimed at providing a pragmatic technique for accurate and precise renal function assessment. The model is incorporated in the online InsightRX precision dosing platform to facilitate clinical application.

We provide an online ready-to-use pharmacometric tool for accurate and precise renal function assessment using three to four blood draws within 3-4 h after iohexol administration. Our pharmacometric tool enables robust renal function evaluation for clinical situations in which current methods show limited accuracy or poor feasibility.

\section{Introduction}

Unequivocal renal function determination is pivotal in many clinical situations. In the renal transplantation setting, this is particularly true for living renal transplant donor eligibility screening and renal transplant function monitoring, as well as for research purposes.

Initial screening of living renal transplant donor candidates and routine monitoring of renal transplant recipients is typically performed with 24-h urinary creatinine clearance or by estimation of the glomerular filtration rate (GFR) using serum creatinine and an estimation formula $\left(\mathrm{eGFR}_{\mathrm{cr}}\right)[1,2]$. Although $\mathrm{eGFR}_{\mathrm{cr}}$ has provided a convenient renal function marker for decades, it shows poor agreement with measured GFR (mGFR) techniques, which are considered to correspond best with the true GFR [3, 4]. However, 24-h urinary creatinine clearance is considered cumbersome owing to the challenge of collecting and transporting the timed urine collection [1]. In donor candidate screening, this poses a challenge when determining the eligibility of donor candidates with borderline 24-h urinary creatinine clearance or eGFR $\mathrm{cr}_{\mathrm{cr}}$, typically within the $60-90 \mathrm{~mL} / \mathrm{min} / 1.73 \mathrm{~m}^{2}$ range $[1,3]$. In recipients, it may render $\mathrm{eGFR}_{\mathrm{cr}}$ to be of limited informative value to monitor transplant function over time [2,3]. International guidelines on donor screening and recipient care acknowledge the limited reliability of $\mathrm{eGFR}_{\mathrm{cr}}$ for these purposes and identify mGFR techniques, which utilise urinary or plasma clearance of exogenous filtration markers, superior in terms of accuracy [1-4]. Although mGFR techniques are generally considered the gold standard for renal function assessment, these can prove burdensome because of their dependency on extensive sampling [3, 5]. This limits the clinical feasibility of mGFR and has hampered its widespread use in routine clinical practice. In recent years, mGFR based on single-dose iohexol plasma clearance has, nonetheless, gained particular clinical interest and is advocated as an alternative for routine renal function evaluation [3-5].

In conventional iohexol mGFR methods, iohexol is administered via a single intravenous bolus injection [6, 7]. Iohexol plasma clearance is then quantified from the full area under the concentration-time curve (AUC), determined by either extensive sampling or sparse sampling during the terminal log-linear elimination phase with subsequent extrapolation to the full AUC using the Brøchner-Mortensen or Jacobsson equation [7-9]. Whereas these methods provide clinically feasible approaches for iohexol GFR determination, they are based on estimations guided by the terminal elimination phase exclusively [6]. Furthermore, these methods continue to rely on extensive sampling or late samples drawn up to $8 \mathrm{~h}$ after iohexol administration $[6,7]$, which still encompasses a large patient burden.

A pharmacometric approach could likely provide a more accurate and robust iohexol GFR estimation, as this technique can capture its entire pharmacokinetic profile. Moreover, it facilitates the development of limited sampling schedules (LSSs) drawn early after iohexol administration to aid clinical application. Indeed, a previously published pharmacometric model showed adequate GFR predictive ability, utilising Bayesian forecasting with four blood samples drawn within $5 \mathrm{~h}$ after iohexol administration [10]. Notably, sampling up to $5 \mathrm{~h}$ was required for adequate estimation reliability for GFRs below $30 \mathrm{~mL} / \mathrm{min}$ [10]. As renal transplant recipients and particularly donor candidates typically show GFRs exceeding $30 \mathrm{~mL} / \mathrm{min}$, this likely allows for application of shorter sampling schemes in this population to further increase iohexol mGFR feasibility.

Here, we aim to develop a population pharmacokinetic model and LSSs for iohexol to provide a pragmatic tool for iohexol GFR determination in the renal transplantation setting. Additionally, we incorporate the model in an 
online precision dosing platform to further aid its clinical application.

\section{Methods}

\subsection{Software}

Data handling, visualisation and statistics were performed in R 3.6.1 (R Foundation for Statistical Computing, Vienna, Austria) and RStudio 1.2.5019 (RStudio Inc., Boston, MA, USA). The pharmacometric analysis was performed with nonlinear mixed-effects modelling software NONMEM ${ }^{\circledR}$ 7.4 (Icon Development Solutions, Ellicott City, MD, USA), using Pirana 2.9.8 and Perl-speaks-NONMEM Toolkit 5.0.0 as a modelling environment $[11,12]$.

\subsection{Pharmacokinetic Data}

The study was based on pooled pharmacokinetic iohexol profiles from 335 renal transplant recipients and 41 living renal transplant donor candidates. These included recipients who participated in the multicentre REPAIR trial (ISRCTN30083294; $n=320$, of which 52 were treated at Leiden University Medical Center [LUMC]) [13] or a phase I study conducted at LUMC (NCT00734396; $n=15$ ) [14], and donor candidates who underwent routine eligibility screening at LUMC $(n=41)$. The ISRCTN30083294 participants received a single intravenous injection of Omnipaque 240 (2590 $\mathrm{mg}$ of iohexol) or Omnipague 300 (3235 $\mathrm{mg}$ of iohexol) with sampling at $5 \mathrm{~min}, 2 \mathrm{~h}, 3 \mathrm{~h}$ and $4 \mathrm{~h}$ after administration, whereas NCT00734396 participants received Omnipaque 300 with sampling at $5 \mathrm{~min}, 1 \mathrm{~h}, 2 \mathrm{~h}, 3 \mathrm{~h}$ and 4 h. Donor candidates received Omnipaque 300 for renal function confirmation purposes, with sampling at $5 \mathrm{~min}, 30 \mathrm{~min}$, $1 \mathrm{~h}, 2 \mathrm{~h}, 2.5 \mathrm{~h}, 3 \mathrm{~h}, 3.5 \mathrm{~h}$ and $4 \mathrm{~h}$. Sex, age, weight, height and the iohexol GFR as determined using the slope-intercept method with the Brøchner-Mortensen correction $\left(\mathrm{GFR}_{\mathrm{bm}}\right)$ [8] were available.

All renal transplant recipients $(n=67)$ and donor candidates $(n=41)$ treated at LUMC were pooled and then split to create a model development and internal validation cohort, using an automated block-randomised assignment of recipients and donor candidates to the development and internal validation cohort in a 2:1 manner. Renal transplant recipients who participated in ISRCTN30083294 but were not treated at LUMC $(n=268)$ were pooled in an external validation cohort.

All data originated from studies with previous medical ethical approval or were collected retrospectively from routine clinical care. Hence, this study by Dutch Law is considered research not subjected to the Medical Research Involving Human Subjects Act. A statement of non-objection was issued by the scientific committee of the Department of Internal Medicine of LUMC (W2019.033). All donor candidates gave written informed consent for retrospective collection of their relevant medical records, in accordance with the European General Data Protection Regulation (GDPR; Regulation [EU] 2016/679).

\subsection{Bioanalytics}

A novel, high-performance liquid chromatography assay combined with ultraviolet detection was developed for quantification of iohexol in plasma for NCT00734396 and routine donor eligibility screening, based on two previous assays $[15,16]$. Details on the technical aspects and analytical validation are provided in the Electronic Supplementary Material (ESM).

Iohexol quantification for ISRCTN30083294 was performed at Evelina London Children's Hospital (London, UK) using a previously validated, high-performance liquid chromatography tandem mass spectrometry assay [17]. No substantial divergence between the iohexol results from LUMC and Evelina London Children's Hospital was expected, as these assays have a similar analytical set-up and both centres participate in interlaboratory proficiency testing (Equalis AB, Uppsala, Sweden) for their iohexol assays. For thoroughness, 24 randomly selected NCT00734396 samples were re-analysed at LUMC. All but one (95.8\%) of the iohexol concentrations quantified at LUMC were within $\pm 15 \%$ of Evelina London Children's Hospital, and 21/24 $(87.5 \%)$ within $\pm 10 \%$. These findings confirmed that the iohexol data from both centres could be applied interchangeably. Further details are provided in the ESM.

\subsection{Pharmacometric Modelling}

\subsubsection{Model Development}

Iohexol pharmacokinetics were estimated from the concentration-time data of the model development cohort using a population pharmacokinetic model. The first-order conditional estimation method with interaction was applied throughout the analysis. Model selection was based primarily on a statistically significant change in the objective function value $(\triangle \mathrm{OFV})$ between a modified model and its precursor, with $\Delta \mathrm{OFV}<-6.64(p<0.01$, degrees of freedom $=1$, assuming $\chi^{2}$ distribution) resulting in selection of the modified model, provided proper model convergence, appropriate visual diagnostics, and acceptable extents of parameter estimate uncertainty and $\eta$-shrinkage $(<30 \%)$ [18]. During base model development, one-, two-, and three-compartmental model structures with linear and first-order elimination were explored and additive, proportional and combined residual error model structures were evaluated. 
A covariate analysis was performed to explore options to optimise the individual predictive performance of the model. Allometric scaling of the parameters to account for between-subject variability (BSV) in body composition was considered likely to improve model performance [19]. Accordingly, we evaluated a covariate model in which all flow and volume parameters were allometrically scaled to a fat-free mass (FFM) of $57.18 \mathrm{~kg}$, corresponding to a male individual with a height of $1.80 \mathrm{~m}$ and total body weight of $70 \mathrm{~kg}$. The FFM was predicted from sex, total bodyweight and height utilising standard equations [20]. All flow parameters in this model were exponentiated by 0.75 , whereas linear proportionality was assumed for volume parameters [21]. In addition, a discrepancy between the typical renal function of renal transplant donor candidates and recipients is apparent. Hence, accounting for this divergence by the addition of patient type ('recipient' or 'donor candidate') as a covariate was considered likely to improve model performance. Characterisation of the final covariate model was guided by biological plausibility, reduction of the random variability, improvement of visual diagnostics, model stability and the $\triangle \mathrm{OFV}$

Graphical model evaluation was performed using standard diagnostic plots [22] and prediction-corrected visual predictive checks (pcVPCs; $n=1000$ ) [23]. As the model was intended primarily for individual clearance estimation from pharmacokinetic measurements using maximum a posteriori Bayesian estimation (MAP-BE), most emphasis was laid on the individual predictive performance of the model. Evaluation of the robustness of the final parameter estimates was performed with the bootstrap procedure in Perl-speaks-NONMEM $(n=1000)$ [24], stratified to patient type to ensure an even distribution of recipients and donor candidates in the resampling datasets.

\subsubsection{Model Validation}

The final model was validated on the internal and external validation cohorts using a two-step approach. First, a model run with the parameter re-estimation option enabled and a bootstrap analysis $(n=1000)$ were performed on the internal validation cohort to evaluate parameter estimate robustness. Second, we evaluated the performance of the model using pcVPCs $(n=1000)$ and individual prediction diagnostics on the internal and external validation cohorts. For the latter analysis, all model parameters were fixed to the median population values of the final model.

Additionally, we compared the model-predicted individual iohexol clearances to the $\mathrm{GFR}_{\mathrm{bm}}$ to assess whether its ability to describe individual iohexol concentrations coincides with reliable GFR predictions. A complicating factor for this comparison, however, is that the model was expected to outperform $\mathrm{GFR}_{\mathrm{bm}}$. Namely, the $\mathrm{GFR}_{\mathrm{bm}}$ assumes a terminal log-linear elimination phase for every patient from $2 \mathrm{~h}$ after administration. Moreover, the mathematical extrapolation to time zero is highly dependent of a correct characterisation of the log-linear regression slope. Furthermore, and most importantly, the Brøchner-Mortensen equation encompasses a correction factor tailored to the iohexol distribution profile of the typical patient, whereas this profile varies across GFRs. Albeit helpful to provide a GFR estimate when characterisation of the non-linear portion of the curve is impossible or infeasible, these intrinsic dependencies and assumptions render the $\mathrm{GFR}_{\mathrm{bm}}$ error prone for individual GFR determination. Alternatively, an appropriate pharmacokinetic model enables characterisation of the entire individual iohexol curve utilising all available pharmacokinetic information, likely yielding more accurate and more precise GFR estimates. Comparison between our model-predicted GFRs and the $\mathrm{GFR}_{\mathrm{bm}}$, however, still is informative. For this purpose, a virtual patient population $(n=1000)$ was created in which FFM and patient type were sampled randomly from a univariate distribution and a binomial distribution with equal probabilities, respectively. The final model with residual error was then applied to simulate 5-h iohexol curves. The simulated iohexol concentrations at $5,15,30,45,60,90,120,150,180,210$ and $240 \mathrm{~min}$ were then used as pharmacokinetic input data to derive modelpredicted individual iohexol clearances. The $\mathrm{GFR}_{\mathrm{bm}}$ was calculated following standard practice; the iohexol dose was divided by the area under the log-linear regression curve of the iohexol concentrations at either 120, 180 and 240 min (3-point $\mathrm{GFR}_{\mathrm{bm}}$ ), 120, 150, 180, 210 and $240 \mathrm{~min}$ (5-point $\mathrm{GFR}_{\mathrm{bm}}$ ), or 120, 150, 180, 210, 240, 270 and 300 min (7-point $\mathrm{GFR}_{\mathrm{bm}}$ ) extrapolated to the concentration at time zero, corrected for the early distribution phase using the Brøchner-Mortensen formula, assuming a terminal loglinear elimination phase $[6,8]$.

Finally, we evaluated the ability of the model to fit iohexol curves beyond $4 \mathrm{~h}$ after administration using standard individual plots. Whereas the 0- to 4-h window captures most of the iohexol AUC for most renal transplant recipients and donor candidates, it is important to evaluate whether the model adequately captures also the remainder of the curve. Iohexol curves up to $24 \mathrm{~h}$ after Omnipaque 300 administration from 11 random subjects $(n=108)$ with GFRs below $40 \mathrm{~mL} / \mathrm{min}$ who participated in the study by Åsberg et al. [10] were kindly provided by Oslo University Hospital. This centre also participates in the Equalis interlaboratory proficiency testing programme, ensuring iohexol data interchangeability.

\subsubsection{Limited Sampling Schedule Selection}

Various LSSs based on one to four blood draws within the first $4 \mathrm{~h}$ after iohexol administration were evaluated. An 
optimal LSS would require as few samples as possible, drawn as early after administration as possible, while ensuring highly accurate and precise iohexol GFR estimation.

The LSS analysis was performed on the virtual patient population created during model validation. This dataset included model-predicted individual iohexol clearances, estimated from concentrations at 5, 15, 30, 45, 60, 90, 120, $150,180,210$ and $240 \mathrm{~min}$. These individual iohexol clearance estimates were considered the reference GFR values $\left(\mathrm{GFR}_{\text {full }}\right)$. For each LSS, the dataset was subsetted to include only the pharmacokinetic data obtained at the time instances included in that particular LSS. Subsequently, the individual iohexol clearance estimates for each LSS $\left(\mathrm{GFR}_{\mathrm{lss}}\right)$ were derived from estimation of the pharmacokinetic curve up to 240 min using MAP-BE. The $G_{F} R_{\text {full }}$ and $G R_{\text {lss }}$ were then compared to evaluate the predictive performance of the model for each LSS.

The predictive performance was expressed with the mean relative prediction error for bias, and the mean relative absolute prediction error for imprecision [25]. In addition, the root mean squared percentage prediction error (RMSE), Pearson correlation coefficient, total deviation index (TDI) [26], concordance correlation coefficient [27] and the percentages of the $\mathrm{GFR}_{\mathrm{lss}}$ within \pm 5 to $\pm 20 \%$ of the $\mathrm{GFR}_{\text {full }}$ $\left(P_{5}-P_{20}\right)$ were assessed to aid interpretation [3].

\subsubsection{Implementation in the InsightRX Framework}

To provide a certified, robust, ready-to-use, and end-user friendly tool for applying our model, the final model was incorporated in the InsightRX Nova framework (InsightRX, San Francisco, CA, USA). Via a license agreement, InsightRX Nova (www.insight-rx.com) is accessible as an online web-application, built around the open-source PKPDsim simulation library for R (www.pkpdsim-docs.com). Based on collected pharmacokinetic measurements and additional patient characteristics, the platform applies MAP-BE for derivation of the individual estimates for the population model parameters. The platform relies on local electronic medical record software integration, or, when this is not operable, manual input of patient characteristics, dosing information, and collected pharmacokinetic measurements. No modelling knowledge or experience is required to operate the tool. Our final pharmacokinetic model was implemented in an InsightRX Nova dosing module for iohexol. InsightRX Nova adheres to ISO13485 (Quality Management for Medical Devices) and its quality procedures require verification of model implementation for numerical accuracy and robustness. Numerical verification against NONMEM was performed for the simulation of iohexol concentration data based on pharmacokinetic parameters, covariates and dosing regimens, as well as for the calculation of individual estimates using MAP-BE based on simulated input data.

\section{Results}

\subsection{Pharmacokinetic Data}

A total of 394 observations from 72 subjects were available for model development. Internal and external validation were performed with 193 and 1044 observations from 36 and 268 subjects, respectively. The demographics of these cohorts are summarised in Table 1.

\subsection{Pharmacometric Modelling}

\subsubsection{Model Development}

Iohexol pharmacokinetics were best described by a twocompartmental model with first order elimination, with BSV on clearance $(C L)$, intercompartmental clearance $(Q)$ and the central $\left(V_{\mathrm{c}}\right)$ and peripheral distribution volumes $\left(V_{\mathrm{p}}\right)$, and a proportional error model. Efforts into fitting the model with a full variance-covariance matrix of random effects showed slight model instability, which was resolved after

Table 1 Characteristics of the model development and internal and external validation cohorts

\begin{tabular}{|c|c|c|c|c|c|c|c|c|c|}
\hline \multirow[t]{2}{*}{ Characteristics } & \multicolumn{3}{|c|}{ Development cohort } & \multicolumn{3}{|c|}{ Internal validation cohort } & \multicolumn{3}{|c|}{ External validation cohort } \\
\hline & $N$ & Median & Range & $N$ & Median & Range & $N$ & Median & Range \\
\hline Total number of patients & 72 & & & 36 & & & 268 & & \\
\hline Patient type (recipient; donor) & $45 ; 27$ & & & $22 ; 14$ & & & $268 ; 0$ & & \\
\hline Gender (male; female) & $32 ; 40$ & & & $17 ; 19$ & & & $193 ; 75$ & & \\
\hline Age (years) & & 58.0 & $19.9-78.3$ & & 58.3 & $24.8-72.5$ & & 47.2 & $19.1-77.0$ \\
\hline Weight (kg) & & 72.8 & $45.0-124$ & & 73.8 & $50.0-99.0$ & & 80.0 & $38.5-133$ \\
\hline Height $(\mathrm{cm})$ & & 171 & $148-204$ & & 170 & $156-186$ & & 175 & $147-196$ \\
\hline $\mathrm{mGFR}\left(\mathrm{mL} / \mathrm{min} / 1.73 \mathrm{~m}^{2}\right)$ & & 67.0 & $27.0-117$ & & 73.0 & $28.8-113$ & & 58.9 & $16.6-104$ \\
\hline
\end{tabular}

$m G F R$ measured glomerular filtration rate as derived from iohexol plasma clearance, calculated using the slope-intercept method with Brøchner-Mortensen correction 
parametrisation of the matrix to include only the covariance between the BSV in $C L, V_{\mathrm{c}}$ and $Q$, but not $V_{\mathrm{p}}$. A onecompartmental model showed a clear misspecification in the early distribution phase, whereas efforts into fitting a three-compartmental model resulted in overparameterisation. Allometric scaling of all flow and volume parameters to FFM yielded a $\triangle \mathrm{OFV}$ of -19.6 , whereas addition of patient type as a covariate on $C L$ and $V_{\mathrm{c}}$ yielded a $\triangle \mathrm{OFV}$ of -14.1 . Combining both in the final covariate model yielded a $\triangle \mathrm{OFV}$ of -45.7 , with reduction of the random variabilities in $C L(34.1 \% \rightarrow 29.8 \%), V_{\mathrm{c}}(42.2 \% \rightarrow 40.4 \%)$, $\mathrm{Q}\left(67.7 \% \rightarrow 61.7 \%\right.$ and $V_{\mathrm{p}}(28.2 \% \rightarrow 23.5 \%)$. The parameter estimates of the base and final model on the development cohort are summarised in Table 2. The NONMEM code for the final model is provided in the ESM.

The goodness-of-fit plots for the final model are depicted in Fig. 1. The individual predicted and observed iohexol concentrations showed excellent agreement across the concentration range (Fig. 1a) with a RMSE of $3.37 \%$. The CWRES showed an even distribution over the individual predicted iohexol concentrations (Fig. 1b) and GFRs (ESM) within acceptable ranges. The population prediction diagnostics indicated adequate model appropriateness (Fig. 1c-e).

The results of the bootstrap analysis of the final covariate model on the development cohort are presented in Table 2. Convergence was successful in $83.0 \%$ of the bootstrap runs.
All median parameter estimates from the bootstrap analysis were within $5 \%$ of the parameter estimates of the final model, indicating good parameter estimate reliability.

\subsubsection{Model Validation}

The final model was validated successfully on the internal and external validation cohorts. The results of the parameter re-estimation and bootstrap analysis of the final model on the internal validation cohort are presented in Table 2 . Convergence was successful in $89.2 \%$ of the bootstrap runs. The parameters as re-estimated on the validation cohort, in general, showed adequate concordance with those estimated on the development cohort. The estimates of the primary model parameters, $C L, V_{\mathrm{c}}, Q$, and $V_{\mathrm{p}}$, and the covariate relationships were within the $95 \%$ confidence intervals of those estimated on the development cohort. For $V_{\mathrm{c}}$ and $Q$ $\mathrm{BSV}$, however, some divergence between the estimates on the development and validation cohorts was apparent. The pcVPC on the internal validation cohort showed adequate overlap for the median iohexol concentrations, but slight BSV overprediction (Fig. 2a). This was, however, deemed acceptable as these deviations did not exceed the $95 \%$ confidence intervals of the 5th and 95th percentiles of the predicted data. Moreover, the individual prediction diagnostics showed excellent model performance with an RMSE

Table 2 Population pharmacokinetic parameter estimates from the base model, final model, and bootstrap analysis of the final model on the development and internal validation cohort

\begin{tabular}{|c|c|c|c|c|c|}
\hline \multirow{3}{*}{ Parameter } & \multicolumn{3}{|c|}{ Development cohort } & \multicolumn{2}{|c|}{ Internal validation cohort } \\
\hline & Base model & Final model & Bootstrap analysis & Final model & Bootstrap analysis \\
\hline & $\begin{array}{l}\text { Estimate (RSE; } \\
\eta \text {-shrinkage) }\end{array}$ & $\begin{array}{l}\text { Estimate (RSE; } \\
\eta \text {-shrinkage) }\end{array}$ & $\begin{array}{l}\text { Median estimate } \\
(95 \% \mathrm{CI})\end{array}$ & $\begin{array}{l}\text { Estimate (RSE; } \\
\eta \text {-shrinkage) }\end{array}$ & $\begin{array}{l}\text { Median estimate } \\
(95 \% \mathrm{CI})\end{array}$ \\
\hline$C L(\mathrm{~L} / \mathrm{h})$ & $4.19(5 \%)$ & $4.07(5 \%)$ & $4.06(3.65-4.48)$ & $4.32(6 \%)$ & $4.32(3.76-4.81)$ \\
\hline$V_{\mathrm{c}}(\mathrm{L})$ & $8.15(6 \%)$ & $8.36(9 \%)$ & $8.30(7.27-9.60)$ & $8.05(6 \%)$ & 8.17 (7.16-9.17) \\
\hline$Q(\mathrm{~L} / \mathrm{h})$ & $6.50(12 \%)$ & $7.71(9 \%)$ & $7.83(6.05-10.1)$ & $9.56(8 \%)$ & $9.54(7.18-11.3)$ \\
\hline$V_{\mathrm{p}}(\mathrm{L})$ & $6.07(5 \%)$ & $6.88(5 \%)$ & $6.94(6.22-7.62)$ & $7.11(6 \%)$ & $7.13(6.37-8.15)$ \\
\hline \multicolumn{6}{|c|}{ Covariate relationships } \\
\hline Patient type on $C L$ & & $0.483(21 \%)$ & $0.487(0.312-0.692)$ & $0.463(24 \%)$ & $0.469(0.270-0.730)$ \\
\hline Patient type on $V_{\mathrm{c}}$ & & $0.342(38 \%)$ & $0.336(0.100-0.609)$ & $0.351(34 \%)$ & $0.314(0.081-0.595)$ \\
\hline \multicolumn{6}{|c|}{ Between-subject variability (CV\%) } \\
\hline$C L$ & $34.1(8 ; 3)$ & $29.8(11 ; 4)$ & $29.1(22.6-36.3)$ & $22.6(17 ; 2)$ & $22.1(14.2-30.0)$ \\
\hline$V_{\mathrm{c}}$ & $42.2(8 ; 5)$ & $40.4(8 ; 5)$ & $39.7(33.1-46.3)$ & $24.1(11 ; 9)$ & $23.1(17.1-30.7)$ \\
\hline$Q$ & $67.7(10 ; 25)$ & $61.7(12 ; 27)$ & $59.8(31.7-76.4)$ & $14.8(51 ; 8)$ & $16.4(3.57-56.0)$ \\
\hline$V_{\mathrm{p}}$ & $28.2(23 ; 27)$ & $23.5(18 ; 30)$ & $23.6(12.9-32.6)$ & $28.4(19 ; 14)$ & $27.1(13.3-37.3)$ \\
\hline \multicolumn{6}{|c|}{ Random residual variability (CV\%) } \\
\hline Proportional error & $5.27(13 ; 31)$ & $5.23(14 ; 30)$ & $5.16(3.84-6.55)$ & $5.19(21 ; 26)$ & $5.05(3.45-7.38)$ \\
\hline Additive error & 0 (FIX) & 0 (FIX) & $0(\mathrm{FIX})$ & $0(\mathrm{FIX})$ & $0(\mathrm{FIX})$ \\
\hline
\end{tabular}

$C I$ confidence interval, $C L$ total body clearance, $C V \%$ coefficient of variation, $F I X$ fixed, $Q$ intercompartmental clearance, $R S E$ relative standard error, $V_{c}$ volume of distribution of the central compartment, $V_{p}$ volume of distribution of the peripheral compartment 


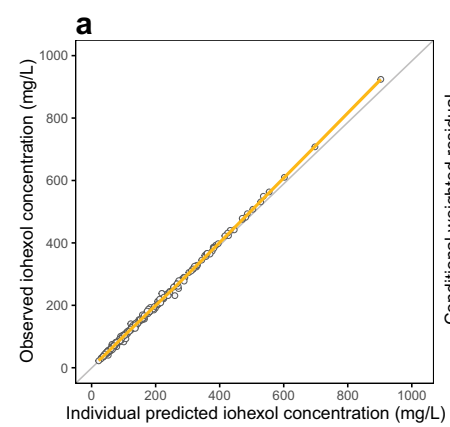

c
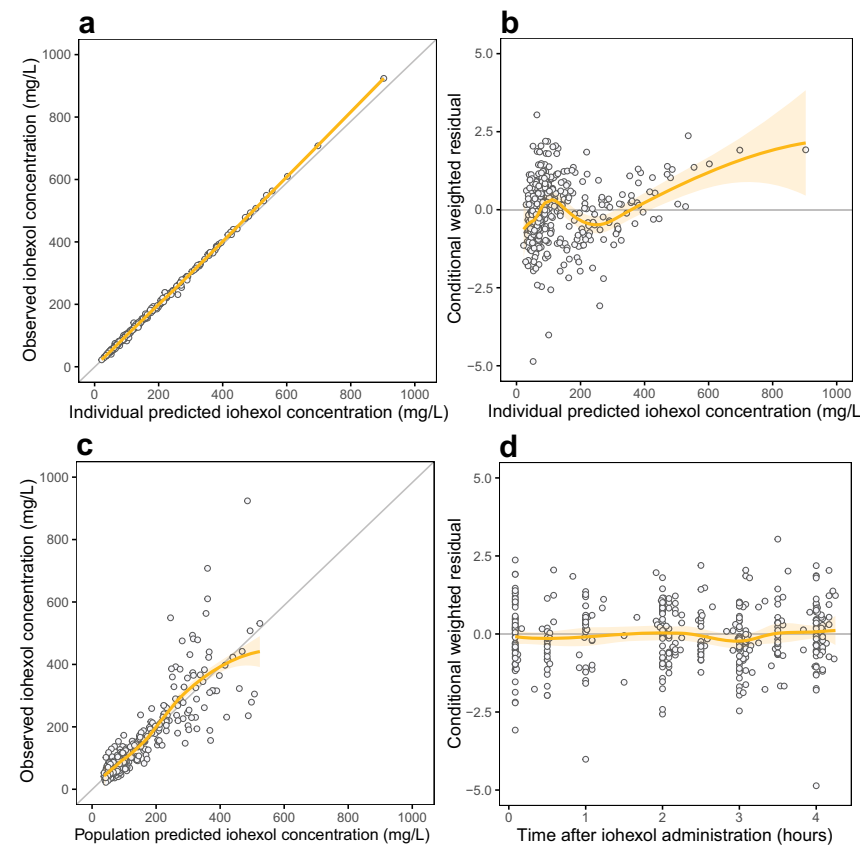

Fig. 1 Diagnostic plots for the final population pharmacokinetic model on the development cohort. a Observed vs individual predicted iohexol concentrations. b Conditional weighted residuals (CWRES) vs individual predicted iohexol concentrations. c Observed vs population predicted iohexol concentrations. d Conditional weighted residuals (CWRES) vs time after iohexol administration. The solid gold lines and gold-shaded areas in a-d represent the local weighted

of $2.68 \%$ between the observed and individual predicted iohexol concentrations (Fig. 2b) and an even distribution of CWRES over the individual predicted iohexol GFRs (ESM). Similarly, the pcVPC on the external validation cohort showed adequate model appropriateness (Fig. 2c), adequate individual prediction diagnostics with a RMSE of 3.79\% (Fig. 2d), and an even distribution of CWRES over the individual predicted iohexol GFRs (ESM). Additionally, although limited data were available for this analysis, the model was able to adequately capture iohexol curves up to $24 \mathrm{~h}$ after administration in patients with impaired renal function (ESM). These findings confirmed that the model is fit for purpose.

Comparison of the model-predicted individual iohexol GFR estimates to the 3-, 5- and 7-point GFR $_{\mathrm{bm}}$ indicated moderate method agreement, with TDI of $18.2-18.7 \%$ and percentage of limited sampling schedule-based GFR predictions within $\pm 10 \%$ of those of the full model $\left(P_{10}\right)$ of 65.0-69.2\% (ESM). The slightly improved method agreement with increasingly informed $\mathrm{GFR}_{\mathrm{bm}}$, however, did indicate a trend towards our model-based GFR estimates. Furthermore, the $\mathrm{GFR}_{\mathrm{bm}}$ is known to incrementally underpredict the GFR beyond approximately $90 \mathrm{~mL} / \mathrm{min}[6,28]$, consistent with its observed incremental underprediction of our model-based GFR predictions beyond $90 \mathrm{~mL} / \mathrm{min}$. Indeed,

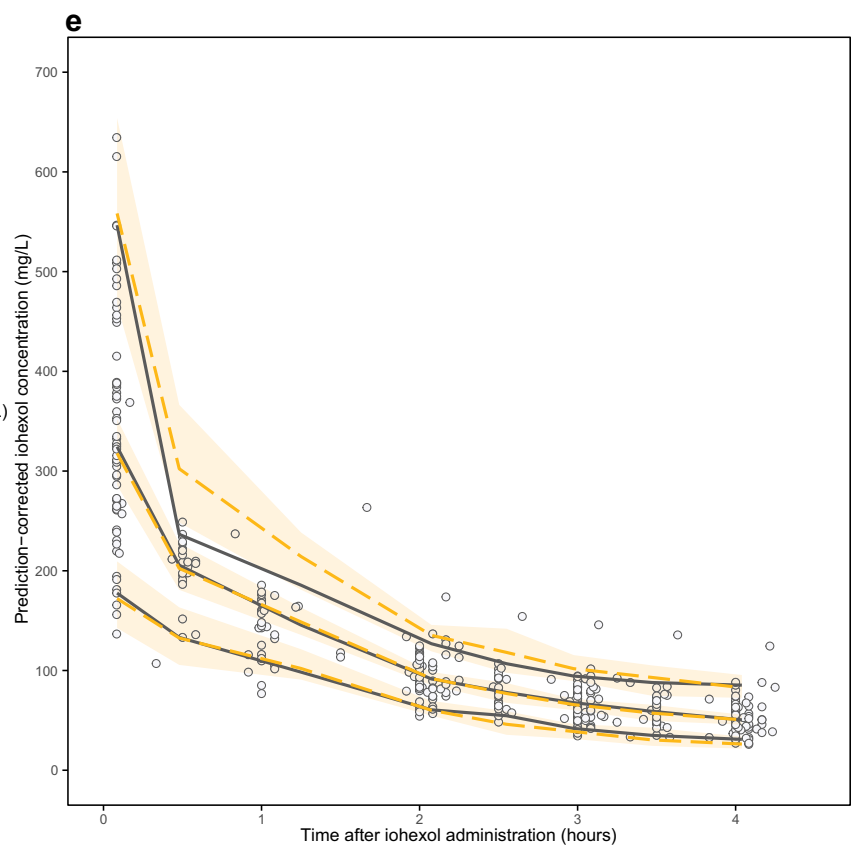

(loess) regression fit and the standard error around the loess regression fit. e Prediction-corrected visual predictive check (VPC), in which the solid black lines represent the 5th, 50th, and 95th percentiles of the observed iohexol concentrations and the dashed gold lines and gold-shaded areas depict the 5th, 50th, and 95th percentiles of the model-simulated iohexol concentrations and their respective $95 \%$ confidence intervals

the $\mathrm{GFR}_{\mathrm{bm}}$ demonstrated considerably higher method agreement for GFRs up to $90 \mathrm{~mL} / \mathrm{min}$, with TDI of $9.47-9.95 \%$ and $P_{10}$ of $75.5-79.9 \%$, indicating acceptable method agreement within the reliable $\mathrm{GFR}_{\mathrm{bm}}$ range. Albeit speculative as the true GFR remains unknown, the residual method disagreement likely provides an estimation of the potential benefit of our model-based approach over the $\mathrm{GFR}_{\mathrm{bm}}$.

\subsubsection{Limited Sampling Schedule Selection}

The predictive performance of all LSSs in terms of bias and precision are depicted in Fig. 3a, b, respectively. Numerical details are provided in the ESM.

Limited sampling schedules based on three to four blood draws within $4 \mathrm{~h}$ after iohexol administration, including one or more early samples and one or more late samples, showed optimal predictive performance with TDI $<7.5 \%$ and $P_{10}>95 \%$. Limited sampling schedules based on three to four blood draws within $3 \mathrm{~h}$ after iohexol administration, in general, showed lower predictive performance. Nevertheless, several of these LSSs still showed a TDI $<10 \%$ and $P_{10}>85 \%$. The best LSSs based on three to four samples within 3-4 h after iohexol administration are highlighted in Table 3, Fig. 4a, b. Two additional LSSs; T5T120T180 and T5T60T120T180, are highlighted because of clinical 
a

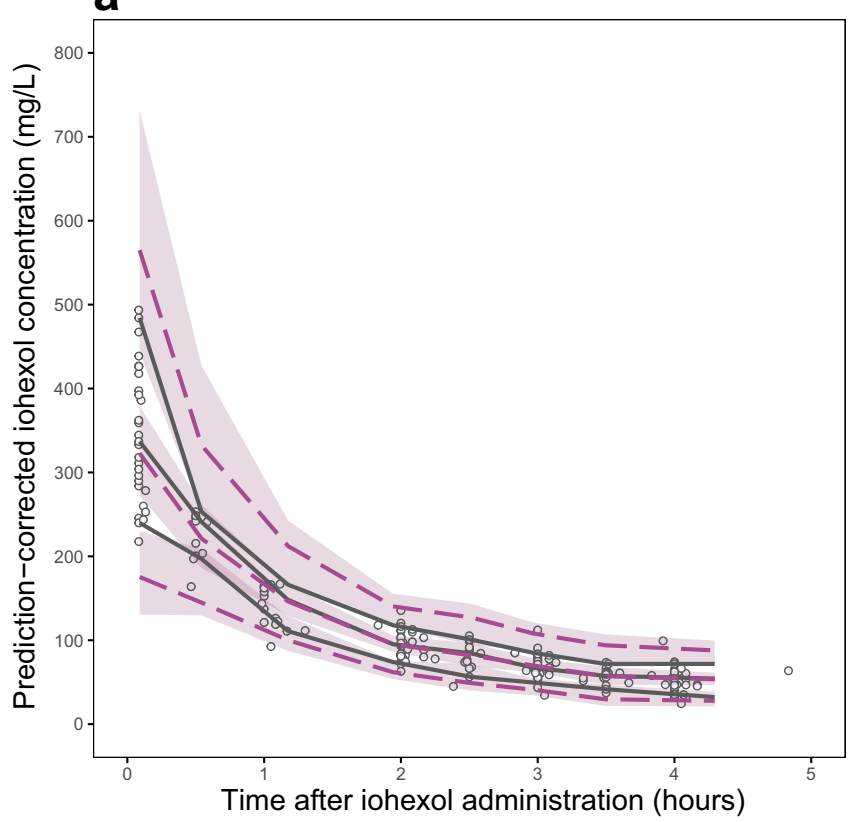

C

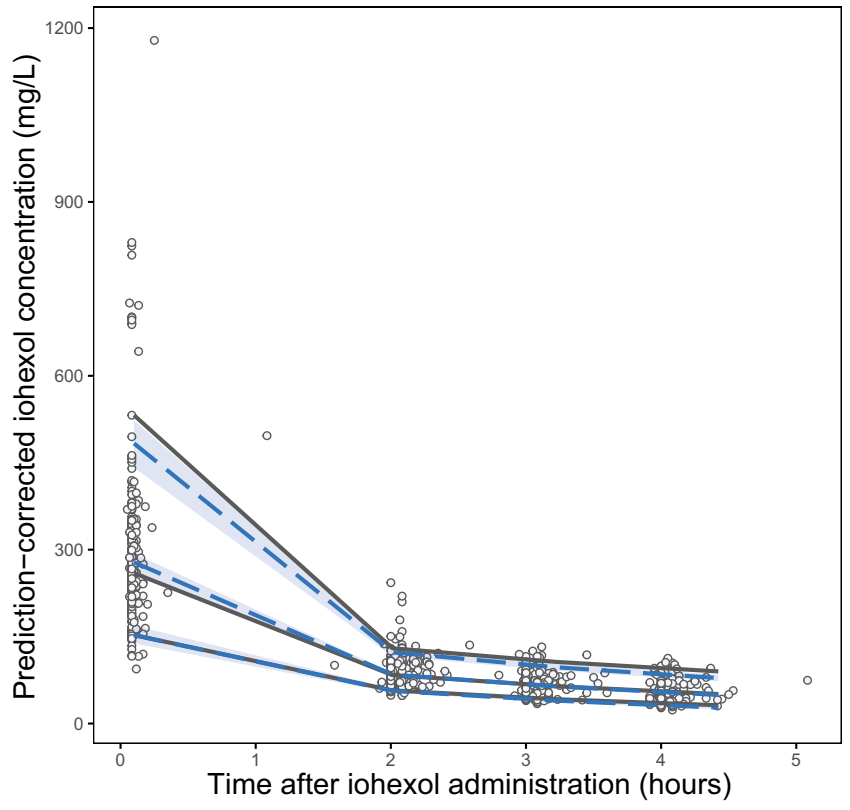

Fig. 2 Prediction-corrected visual predictive checks and individual prediction diagnostic plots for the $\mathbf{a}, \mathbf{b}$ internal validation cohort and $\mathbf{c}, \mathbf{d}$ external validation cohort. The solid black lines in $\mathbf{a}$ and $\mathbf{c}$ represent the 5th, 50th, and 95th percentiles of the observed iohexol concentrations and the dashed purple and blue lines and purple- and

interest. These LSSs could provide options for blood draw alignment with tacrolimus and mycophenolate pharmacometric model-based exposure monitoring in renal transplant recipients, which rely on blood draws pre-dose and at $1 \mathrm{~h}$ (mycophenolate), $2 \mathrm{~h}$ and $3 \mathrm{~h}$ post-dose [29]. The T5T60T120T180 (TDI: $\left.11.5 \% \pm 0.74 \% ; P_{10}: 81.9 \%\right)$ LSS b
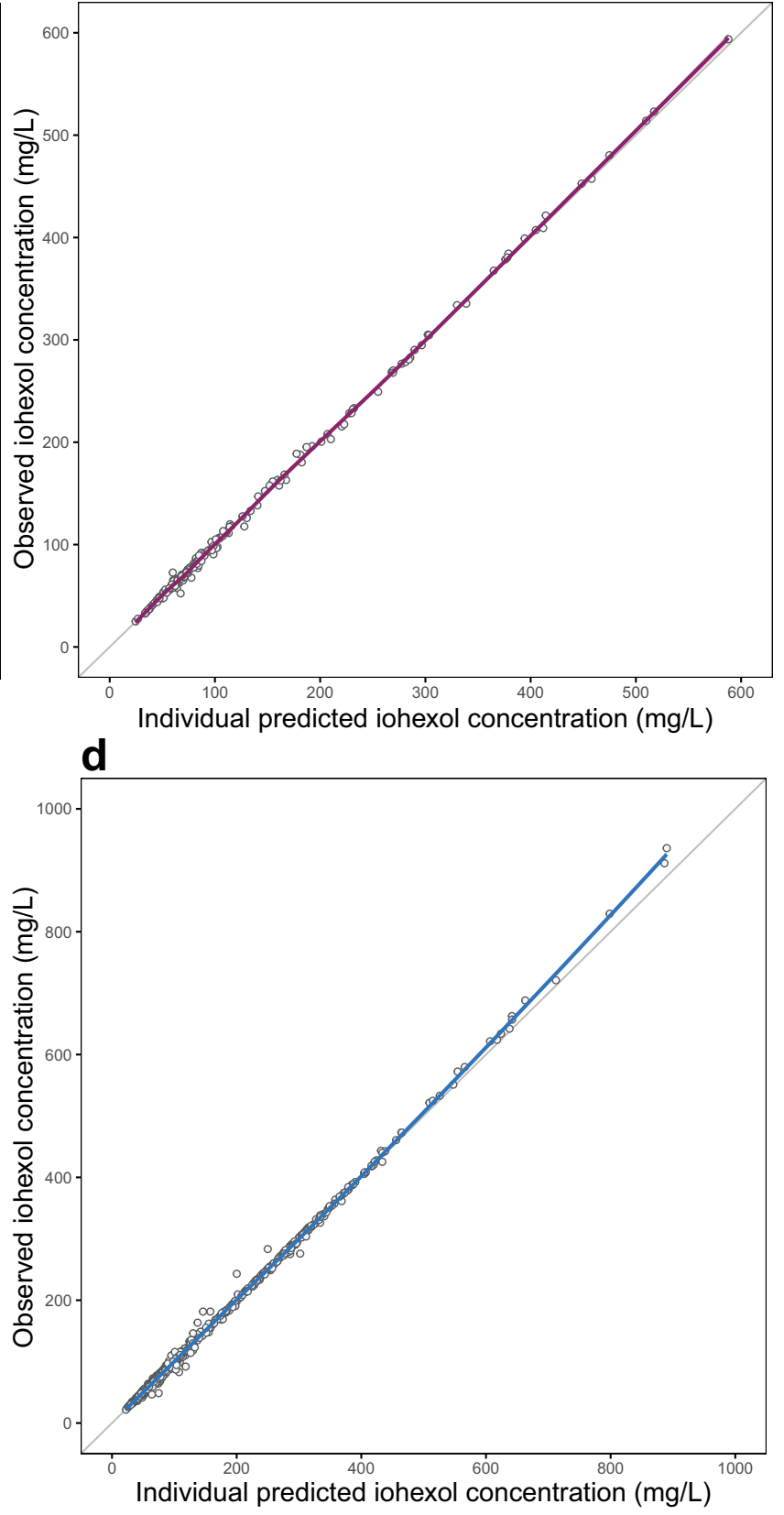

blue-shaded areas depict the 5th, 50th, and 95th percentiles of the model-simulated iohexol concentrations and their respective 95\% confidence intervals. The solid purple and blue lines and purple- and blue-shaded areas in $\mathbf{b}$ and $\mathbf{d}$ represent the local weighted (loess) regression fit and the standard error around the loess regression fit

and T5T120T180 (TDI: $13.8 \% \pm 0.74 \% ; P_{10}: 71.9 \%$ ) showed slightly lower predictive performance as compared to the best LSSs within $3 \mathrm{~h}$ after iohexol administration. Additionally, a previously published LSS with blood draws at 10, 30,120 and $300 \mathrm{~min}$ [10] was evaluated to investigate any potential benefit of sampling up to $5 \mathrm{~h}$ in our population. 


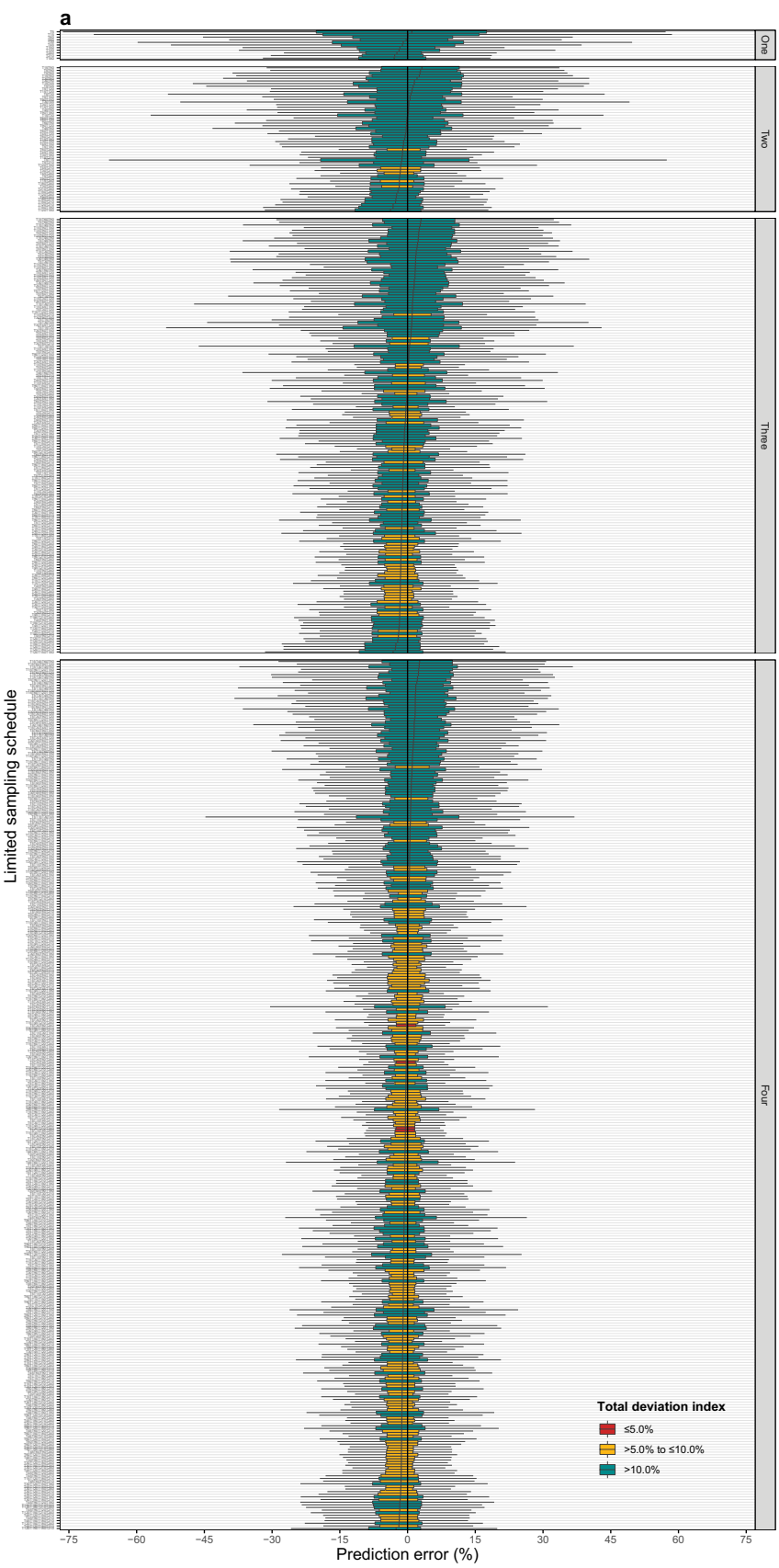

Fig. 3 Limited sampling schedule selection. a Individual iohexol clearance prediction bias of all limited sampling schedules, sorted according to the median bias and the number of sampling instances. b Individual iohexol clearance prediction imprecision, sorted according to the median imprecision and the number of sampling instances.

This LSS showed similar predictive performance as our best LSSs with blood draws up to $4 \mathrm{~h}$, with a TDI and $P_{10}$ of $5.2 \%$ and $97.1 \%$, respectively.

Additionally, albeit beyond their intended application, the performances of the best applicable LSSs were evaluated in 11 patients with impaired renal function using individual

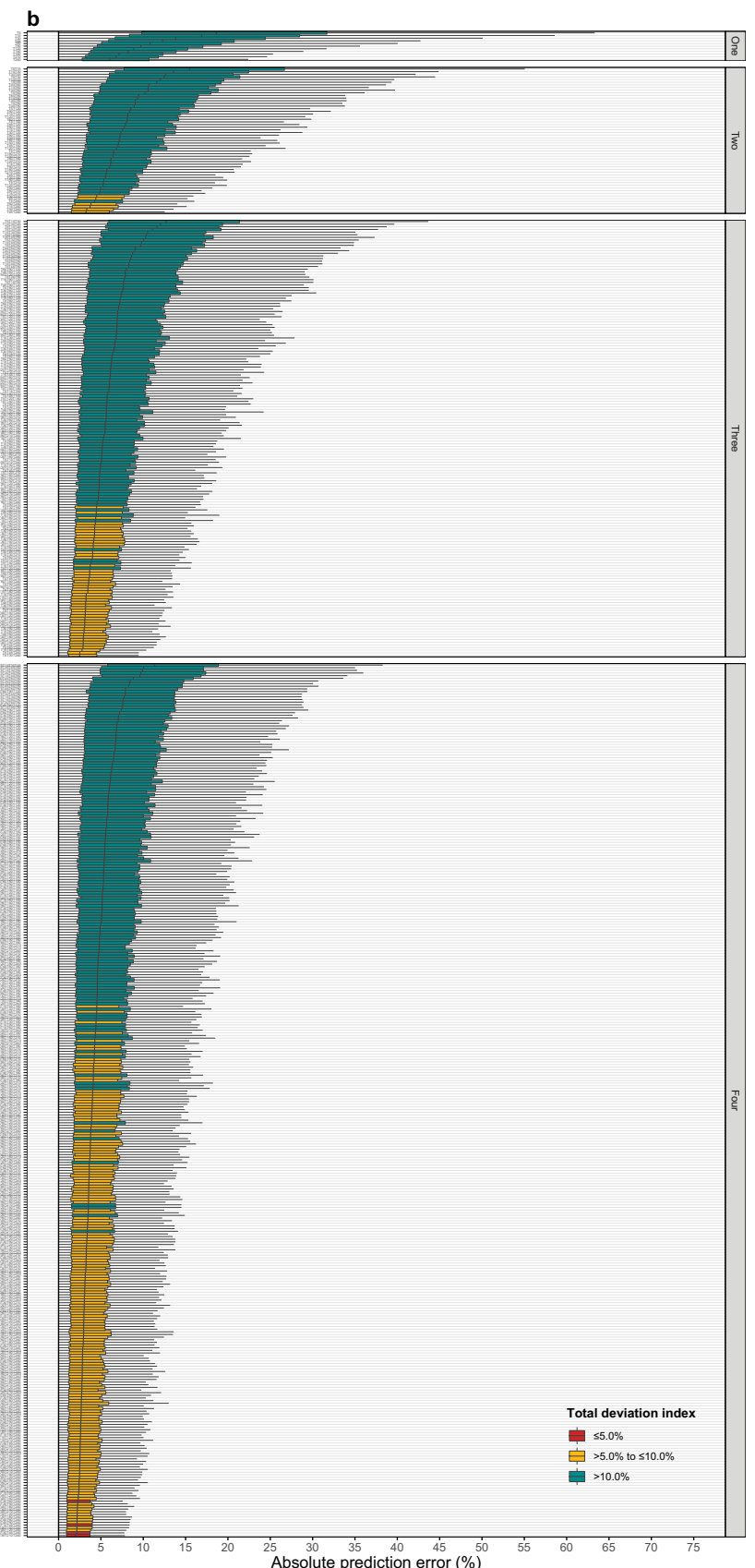

Each boxplot represents the data of a 1000 simulated individuals. Limited sampling schedules that showed a total deviation index (TDI) below $10 \%$ and below $5 \%$, indicating good and excellent predictive performance, are highlighted in gold and red, respectively

plots with the iohexol GFR as determined by Åsberg et al. [10] as reference (ESM). This confirmed that, although the model adequately captures these curves when provided with extensive pharmacokinetic information, it yields biased estimates in patients with GFRs below $30 \mathrm{~mL} / \mathrm{min}$ when relying on limited pharmacokinetic information. Furthermore, 
Fig. 4 Predictive performances of selected limited sampling schedules (LSSs). a Scatter and b Bland-Altman ratio plots of the reference $\left(\mathrm{GFR}_{\text {full }}\right)$ and predicted iohexol clearance $\left(\mathrm{GFR}_{\mathrm{lss}}\right)$. The best LSSs based on three to four samples within $3 \mathrm{~h}$ are depicted in gold, whereas the two LSSs with particular clinical interest are depicted in purple. The best LSSs based on three to four samples within $4 \mathrm{~h}$ are indicated in blue. Solid gold, purple, and blue lines and gold-, purple-, and blue-shaded areas represent the loess regression fits and their standard errors. The solid and upper and lower dashed black lines represent the mean ratios, upper limits of agreement (LoA) and lower LoA. Solid grey lines represent the lines of equality

it stresses the need for the application of 4-point LSSs up to $4 \mathrm{~h}$ in patients with GFRs in the range of $30-40 \mathrm{~mL} / \mathrm{min}$.

Finally, as MAP-BE is guided predominantly by pharmacokinetic input, exclusion of covariate information may be considered to enhance clinical feasibility. When relying on limited pharmacokinetic input, however, this may impair the predictive performance of the model. Hence, three reduced versions of the final model were created to assess whether FFM (Model 2), patient type (Model 3) or both (Model 4) could be excluded from the final model (Model 1). Models 2-4 were fitted to the development dataset for parameter reestimation, fixed, applied to the LSS dataset with the four best LSSs and evaluated for their predictive performance. Models 2-4 showed reduced predictive performance for all four LSSs, with $0.03-1.02 \%$ higher TDI and $0.20-5.60 \%$ lower $P_{10}$ as compared to Model 1. Covariate information thus is essential to ensure adequate LSS performance. Details are provided in the ESM.

\subsubsection{Implementation in the InsightRX Platform}

The final model was implemented successfully in the InsightRX Nova platform. Using simulated data for a virtual patient population, all simulated concentration data and all estimated individual pharmacokinetic parameters were within $1 \%$ and $5 \%$ of those estimated by NONMEM, respectively. All essential pharmacokinetic parameters, in this case, iohexol clearance, were within $1 \%$ of those estimated by NONMEM. The iohexol dosing module was made available in the InsightRX Nova platform. The verification report and user interface are provided in the ESM.

\section{Discussion}

A population pharmacokinetic model and LSSs for iohexol mGFR estimation in renal transplant recipients and living renal transplant donor candidates were developed. Our approach enables pragmatic mGFR determination for donor candidate eligibility screening and renal transplant function monitoring for clinical and research purposes. This is the first study to describe the population pharmacokinetics of 

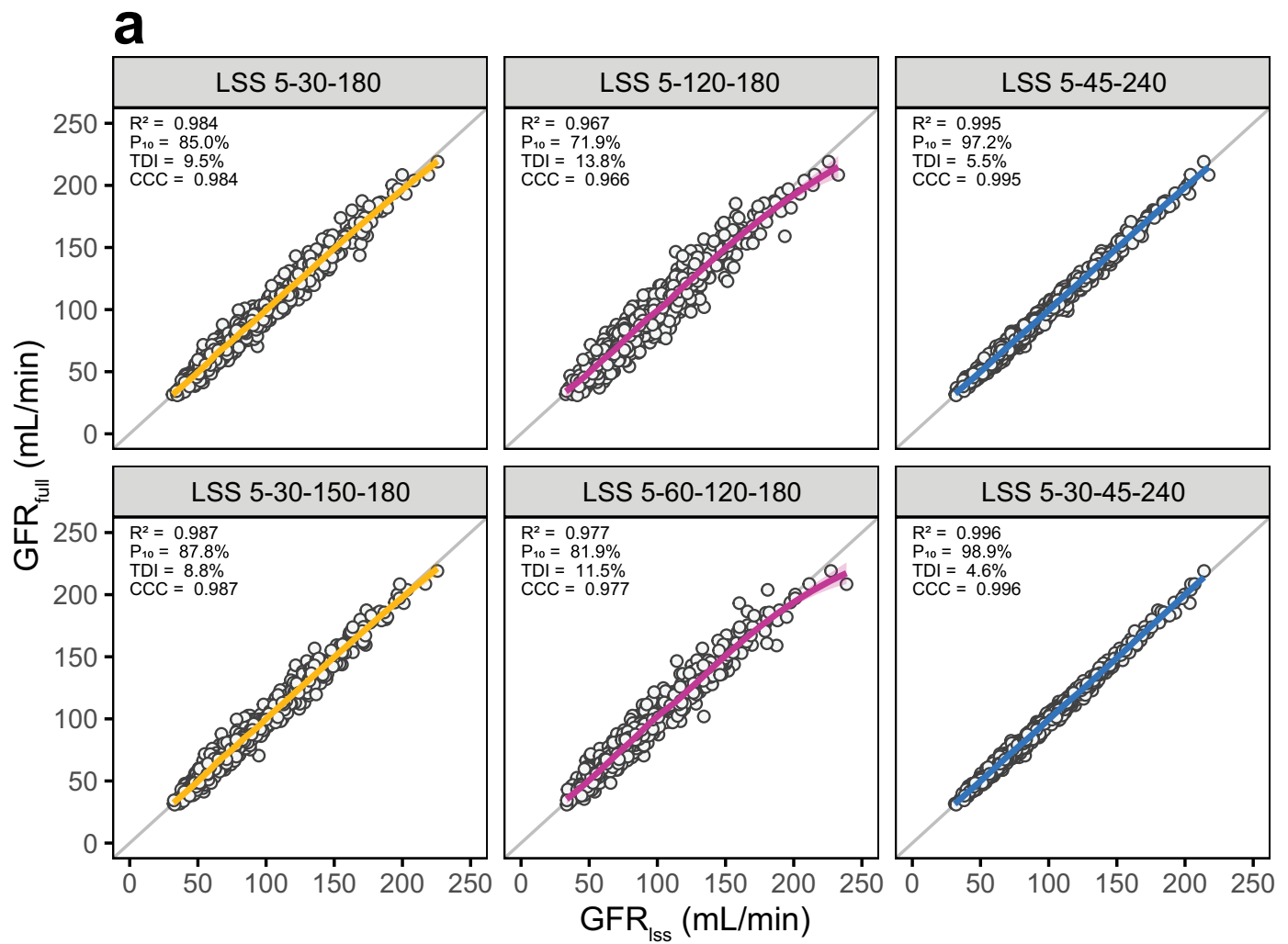

\section{b}

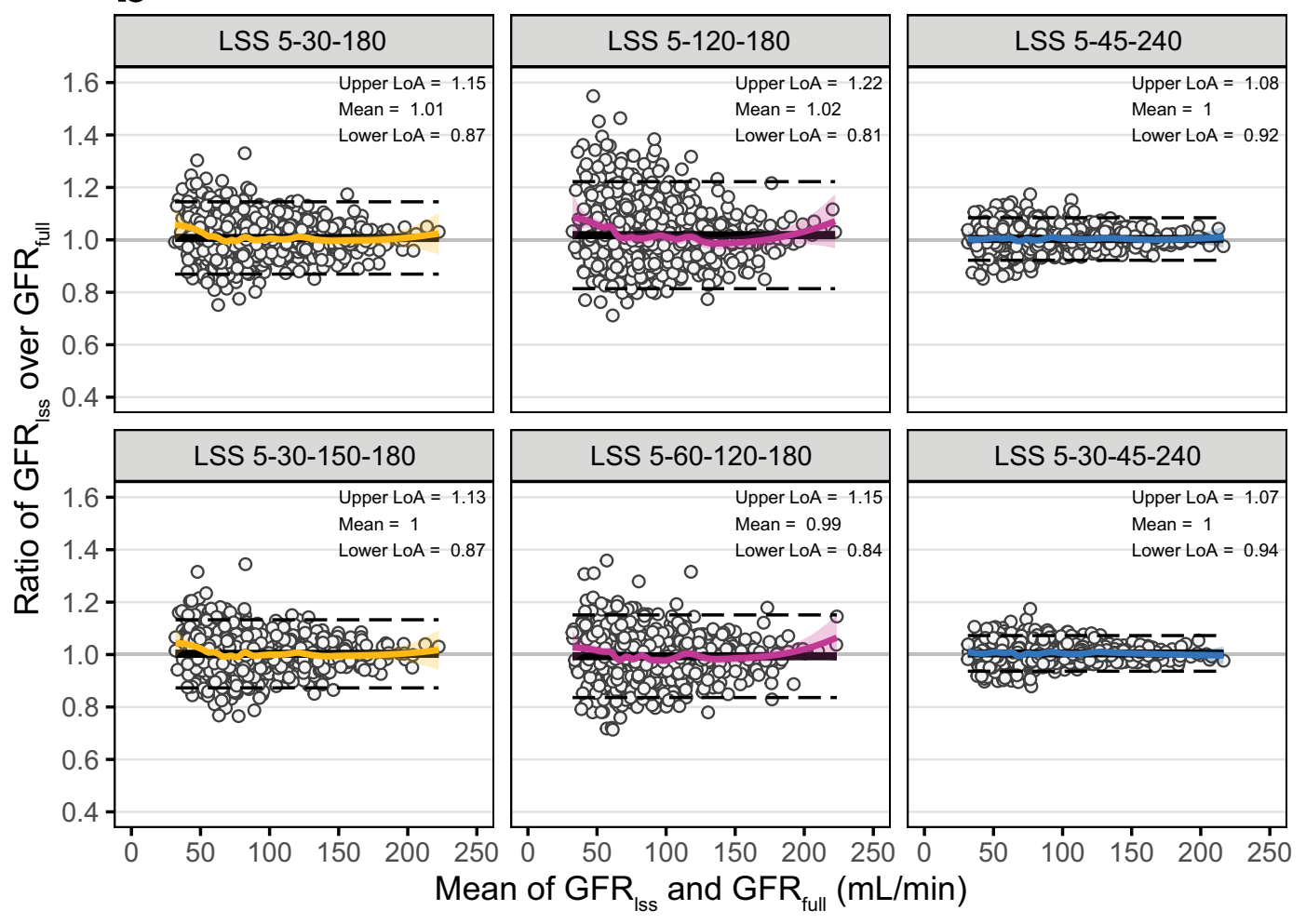


iohexol in donor candidates and the third for recipients [30, 31]. Furthermore, the study provides clinically feasible LSSs based on three to four blood draws within 3-4 h after iohexol administration, whereas previous studies reported LSSs that relied on sampling up to $4.5-5 \mathrm{~h}$ [10, 31]. Finally, we implemented our model in a validated point-of-care precision dosing platform, which facilitates its application in clinical practice, especially when integrated with local electronic medical record software.

A two-compartmental model adequately captured the concentration-time data, showing good resemblance between individual predicted and measured iohexol concentrations in the development and validation cohorts. Allometric scaling of all flow and volume parameters to FFM and the inclusion of patient type as a covariate on $C L$ and $V_{\mathrm{c}}$ improved the model and its individual predictions. During internal validation, some divergence regarding the BSVs of $Q$ and $V_{\mathrm{c}}$, was apparent. The individual predictive performance combined with good stability of $C L$ and $V_{\mathrm{c}}$ did, however, provide reassurance that the model is fit for purpose, which was confirmed in the external validation. Comparison of our model-predicted GFR against the $\mathrm{GFR}_{\mathrm{bm}}$ showed adequate method agreement in the $30-90 \mathrm{~mL} / \mathrm{min}$ range. Agreement in the higher GFR range was moderate, likely explained by incremental GFR underprediction of the $\mathrm{GFR}_{\mathrm{bm}}$ at higher GFRs $[6,28]$.

Previously published population pharmacokinetic models for iohexol comprised one-compartmental [30], two-compartmental $[10,31,32]$ and three-compartmental models [33]. Taubert et al. showed that their initial two-compartmental population model based on data from 570 elderly patients displayed underprediction in the early distribution phase, which was resolved in a three-compartmental model [33]. Efforts into fitting a three-compartmental model to our data, however, resulted in overparameterisation. Benzde Bretagne et al. developed a one-compartmental model in 95 renal transplant recipients, but this model described only the terminal log-linear elimination phase and still relied on Brøchner-Mortensen extrapolation [30]. Riff et al. developed a model in 151 renal transplant recipients with pharmacokinetic data up to $4.5 \mathrm{~h}$ after administration, and developed 3-point LSSs with data from 8 to 22 patients [31]. Notably, their LSSs were not validated for patients with GFRs below $30 \mathrm{~mL} / \mathrm{min}$ [31]. Moreover, the limited number of patients complicates interpretation of their overall validity [31]. Åsberg et al. developed their model in 219 patients with pharmacokinetic data up to $24 \mathrm{~h} \mathrm{[10].} \mathrm{Their} \mathrm{4-point}$ LSS within $5 \mathrm{~h}$ showed excellent predictive performance for GFRs of 14-149 mL/min, whereas a 3-h LSS showed unacceptable extents of bias and imprecision in the lower GFR range [10]. Application of their 5-h LSS with our model yielded similar predictive performance as observed for our 4-h LSSs, confirming that deprecated sampling up to $4 \mathrm{~h}$ is possible without impairing LSS performance in our population with GFRs exceeding $30 \mathrm{~mL} / \mathrm{min}$. Ultimately, selection of an appropriate model and LSS should be guided by the trade-off between clinical pragmatism and LSS predictive performance, on which considerations may vary across clinical situations and centres.

Our study showed some limitations. First, most pharmacokinetic data originated from subjects with estimated GFRs between 30 and $150 \mathrm{~mL} / \mathrm{min}$, drawn up to $4 \mathrm{~h}$ after iohexol administration. Whereas most of the iohexol AUC is captured within this 4-h time frame for most of these patients, the model would ideally have been based on full iohexol pharmacokinetic profiles from subjects across the entire GFR range. Unfortunately, limited data from patients with GFRs below $30 \mathrm{~mL} / \mathrm{min}$ were available, thwarting model and LSS development for this GFR range. This warrants adaptation and validation of our model and LSS for renal transplant recipients with a GFR $<30 \mathrm{~mL} / \mathrm{min}$ before considering this technique for these patients, which could be valuable for clinical decisions concerning dialysis and medication and dose adaptation. In contrast, the model and LSSs were successfully developed and validated for GFRs between 30 and $150 \mathrm{~mL} / \mathrm{min}$, which captures most renal transplant recipients and living renal transplant donor candidates. Moreover, the majority of donor eligibility decisions and longitudinal renal transplantation research, and part of renal functionguided clinical decisions regarding medication and dose adaptation, for which mGFR determination shows particular added value over conventional eGFR assessment, occur in subjects with GFRs exceeding $30 \mathrm{~mL} / \mathrm{min}$. This limitation thus only slightly narrows the added value of our technique. Second, our approach assumes iohexol to be fully cleared renally, whereas a small portion of iohexol $(<5 \%)$ undergoes non-renal excretion [4]. Additional validation against, for instance, urinary inulin clearance could have provided additional insight in the accuracy of our approach. Third, small deviations between the prescribed iohexol doses and the actually administered doses may have occurred, as the syringes were not weighed before and after administration as suggested previously [10]. This may have contributed to the residual error, although the contributions of measurement errors and small sampling time reporting deviations, especially early after iohexol administration, are likely more important. Fourth, part of our study was based on simulations beyond $4 \mathrm{~h}$ after iohexol administration. As the model was developed and thoroughly validated using pharmacokinetic data up to $4 \mathrm{~h}$ exclusively, simulations beyond $4 \mathrm{~h}$ may be associated with additional uncertainty. This uncertainty, however, is likely limited given the rather standard terminal log-linear clearance of iohexol, and the demonstrated ability of the model to adequately fit curves beyond $4 \mathrm{~h}$. Fifth, InsightRX is only accessible with a license, which may pose a hurdle for applying our tool as compared to open-source 
solutions. In contrast, such a professional platform guarantees a certified and validated tool with sustained end-user support, adequate maintenance, data safety and flexibility to adapt to local situations, whereas open-source solutions are not seldomly short-lived owing to insufficient maintenance, limited end-user support and low flexibility. Moreover, clinical laboratory accreditation requirements demand such dosing tools to be certified and validated, which poses an important hurdle for realising open-source solutions. Last, it is important to acknowledge that mGFR, including urinary inulin clearance, also introduces bias in comparison to the true GFR and that outcomes may vary across markers and laboratory sites [34].

Additional possibilities to aid the clinical application of iohexol mGFR in renal transplant recipients include microsampling. We have previously developed a volumetric dried blood spot method for remote tacrolimus and mycophenolic acid exposure monitoring [29]. As tacrolimus and mycophenolate exposure are estimated using the trough, 1-, 2- and 3-h concentrations [29], the T5T60T120T180 iohexol LSS could allow for blood draw alignment. Incorporation of iohexol in the multiplex immunosuppressant assay could then allow for simultaneous (partially) remote renal function and immunosuppressant monitoring. This may provide options for further reducing patient burden and costs, as patients would only have to come to the outpatient clinic for iohexol administration and the first sample and perform the remaining samples remotely. Furthermore, remote microsampling may enable pragmatic extended iohexol sampling for patients with impaired renal function.

Last, our approach could be interesting for application in other populations. As living renal transplant donor candidates are mostly healthy individuals across the adult age range, it seems valid to apply the model and LSSs for renal evaluation in healthy volunteers as these generally show similar renal functions. This could particularly be valuable for research purposes [4]. Healthy volunteer populations, however, usually show an overrepresentation of young male individuals, which may warrant external model validation.

\section{Conclusions}

The developed pharmacometric tool and LSSs with three to four blood draws within 3-4 h after iohexol administration provide an accurate, robust and pragmatic approach for iohexol mGFR assessment in the renal transplantation setting. This technique can be readily implemented in routine clinical care and fills a clear gap for clinical situations in which conventional renal function evaluation methods lack accuracy or show limited feasibility. Additional adaptation and validation of our model and LSSs for renal transplant recipients with GFRs $<30 \mathrm{~mL} / \mathrm{min}$ are warranted before considering this technique in these patients.

Supplementary Information The online version contains supplementary material available at https://doi.org/10.1007/s40262-021-00998-z.

Acknowledgements The authors thank Anders Åsberg and Stein Bergan of Oslo University Hospital for contributing data to this work, and appreciate the assistance of the analytical staff of the Department of Clinical Pharmacy and Toxicology of LUMC.

\section{Declarations}

Funding No funding was received for the preparation of this article.

Conflict of interest Ron Keizer is an employee and stockholder of InsightRX. Tom Zwart, Aiko de Vries, Aline Engbers, Ruth Dam, Paul van der Boog, Jesse Swen, Neil Dalton, Henk-Jan Guchelaar, Johan de Fijter and Dirk Jan Moes have no conflicts of interest that are directly relevant to the content of this article.

Ethics approval All data for this study originated from participants from studies with previous medical ethical approval or were collected retrospectively from routine clinical care. Hence, this study by Dutch Law is considered research not subjected to the Medical Research Involving Human Subjects Act. A statement of non-objection was issued by the scientific committee of the Department of Internal Medicine of LUMC (W2019.033).

Consent to participate All routinely screened donor candidates gave written informed consent for retrospective collection of their relevant medical records, in accordance with the European General Data Protection Regulation (Regulation [EU] 2016/679). The Scientific Committee of the Department of Internal Medicine of LUMC determined that participants from studies with previous medical ethical did not need to give additional written informed consent for retrospective use of their data for this study.

Consent for publication Not applicable.

Data availability The datasets generated during and/or analysed during this study are available from the corresponding author upon reasonable request.

Code availability The NONMEM code used to generate and/or analyse the data described in this study is provided in the ESM.

Author contributions TCZ, APJdV, H-JG, JWdF and DJARM contributed to the study conception and design. TCZ and DJARM performed the research and analysed the data. RND and REvD contributed data. RJK contributed analytical tools. The initial draft of the manuscript was written by TCZ, APJdV and DJARM and all authors commented on previous versions of the manuscript. All authors read and approved the final version of the manuscript.

Open Access This article is licensed under a Creative Commons Attribution-NonCommercial 4.0 International License, which permits any non-commercial use, sharing, adaptation, distribution and reproduction in any medium or format, as long as you give appropriate credit to the original author(s) and the source, provide a link to the Creative Commons licence, and indicate if changes were made. The images or other third party material in this article are included in the article's Creative 
Commons licence, unless indicated otherwise in a credit line to the material. If material is not included in the article's Creative Commons licence and your intended use is not permitted by statutory regulation or exceeds the permitted use, you will need to obtain permission directly from the copyright holder. To view a copy of this licence, visit http://creativecommons.org/licenses/by-nc/4.0/.

\section{References}

1. Lentine KL, Kasiske BL, Levey AS, Adams PL, Alberú J, Bakr MA, et al. KDIGO clinical practice guideline on the evaluation and care of living kidney donors. Transplantation. 2017;101(8S Suppl. 1):S1-109.

2. Kidney Disease: Improving Global Outcomes (KDIGO) Transplant Work Group. KDIGO clinical practice guideline for the care of kidney transplant recipients. Am J Transplant. 2009;9(Suppl. 3):S1-155.

3. Porrini E, Ruggenenti P, Luis-Lima S, Carrara F, Jiménez A, de Vries APJ, et al. Estimated GFR: time for a critical appraisal. Nat Rev Nephrol. 2019;15(3):177-90.

4. Delanaye P, Melsom T, Ebert N, Bäck SE, Mariat C, Cavalier E, et al. Iohexol plasma clearance for measuring glomerular filtration rate in clinical practice and research: a review. Part 2: why to measure glomerular filtration rate with iohexol? Clin Kidney J. 2016;9(5):700-4.

5. Soveri I, Berg UB, Björk J, Elinder CG, Grubb A, Mejare I, et al. Measuring GFR: a systematic review. Am J Kidney Dis. 2014;64(3):411-24.

6. Delanaye P, Ebert N, Melsom T, Gaspari F, Mariat C, Cavalier E, et al. Iohexol plasma clearance for measuring glomerular filtration rate in clinical practice and research: a review. Part 1: how to measure glomerular filtration rate with iohexol? Clin Kidney J. 2016;9(5):682-99.

7. Carrara F, Gaspari F. GFR measured by iohexol: the best choice from a laboratory perspective. J Lab Precis Med. 2018;3:77.

8. Brøchner-Mortensen J. A simple method for the determination of glomerular filtration rate. Scand J Clin Lab Invest. 1972;30(3):271-4.

9. Jacobsson L. A method for the calculation of renal clearance based on a single plasma sample. Clin Physiol. 1983;3(4):297-305.

10. Åsberg A, Bjerre A, Almaas R, Luis-Lima S, Robertsen I, Salvador CL, et al. Measured GFR by utilizing population pharmacokinetic methods to determine iohexol clearance. Kidney Int Rep. 2020;5(2):189-98.

11. Keizer RJ, van Benten M, Beijnen JH, Schellens JH, Huitema AD. Pirana and PCluster: a modeling environment and cluster infrastructure for NONMEM. Comput Methods Programs Biomed. 2011;101(1):72-9.

12. Lindbom L, Pihlgren P, Jonsson EN. PsN-Toolkit: a collection of computer intensive statistical methods for non-linear mixed effect modeling using NONMEM. Comput Methods Programs Biomed. 2005;79(3):241-57.

13. MacAllister R, Clayton T, Knight R, Robertson S, Nicholas J, Motwani M, et al. REmote preconditioning for Protection Against Ischaemia-Reperfusion in renal transplantation (REPAIR): a multicentre, multinational, double-blind, factorial designed randomised controlled trial. Southampton: NIHR Journals Library; 2015.

14. Reinders ME, de Fijter JW, Roelofs H, Bajema IM, de Vries DK, Schaapherder AF, et al. Autologous bone marrow-derived mesenchymal stromal cells for the treatment of allograft rejection after renal transplantation: results of a phase I study. Stem Cells Transl Med. 2013;2(2):107-11.

15. Gaspari F, Perico N, Ruggenenti P, Mosconi L, Amuchastegui CS, Guerini E, et al. Plasma clearance of nonradioactive iohexol as a measure of glomerular filtration rate. J Am Soc Nephrol. 1995;6(2):257-63.

16. Gaspari F, Guerini E, Perico N, Mosconi L, Ruggenenti P, Remuzzi G. Glomerular filtration rate determined from a single plasma sample after intravenous iohexol injection: is it reliable? J Am Soc Nephrol. 1996;7(12):2689-93.

17. Dixon JJ, Lane K, Dalton RN, Turner C, MacPhee IAM, Chis Ster I, et al. Continuous infusion of low-dose Iohexol measures changing glomerular filtration rate in critically ill patients. Crit Care Med. 2018;46(3):e190-7.

18. Savic RM, Karlsson MO. Importance of shrinkage in empirical Bayes estimates for diagnostics: problems and solutions. AAPS J. 2009;11(3):558-69.

19. Holford NHG, Anderson BJ. Allometric size: the scientific theory and extension to normal fat mass. Eur J Pharm Sci. 2017;15(109s):S59-64.

20. Janmahasatian S, Duffull SB, Ash S, Ward LC, Byrne NM, Green B. Quantification of lean bodyweight. Clin Pharmacokinet. 2005;44(10):1051-65.

21. West GB, Brown JH, Enquist BJ. A general model for the origin of allometric scaling laws in biology. Science. 1997;276(5309):122-6.

22. Nguyen TH, Mouksassi MS, Holford N, Al-Huniti N, Freedman I, Hooker AC, et al. Model evaluation of continuous data pharmacometric models: metrics and graphics. CPT Pharmacometrics Syst Pharmacol. 2017;6(2):87-109.

23. Bergstrand M, Hooker AC, Wallin JE, Karlsson MO. Prediction-corrected visual predictive checks for diagnosing nonlinear mixed-effects models. AAPS J. 2011;13(2):143-51.

24. Yafune A, Ishiguro M. Bootstrap approach for constructing confidence intervals for population pharmacokinetic parameters. I: a use of bootstrap standard error. Stat Med. 1999;18(5):581-99.

25. Sheiner LB, Beal SL. Some suggestions for measuring predictive performance. J Pharmacokinet Biopharm. 1981;9(4):503-12.

26. Lin LI. Total deviation index for measuring individual agreement with applications in laboratory performance and bioequivalence. Stat Med. 2000;19(2):255-70.

27. Lin LI. A concordance correlation coefficient to evaluate reproducibility. Biometrics. 1989;45(1):255-68.

28. De Sadeleer C, Piepsz A, Ham HR. How good is the slope on the second exponential for estimating 51Cr-EDTA renal clearance? A Monte Carlo simulation. Nucl Med Commun. 2000;21(5):455-8.

29. Zwart TC, Gokoel SRM, van der Boog PJM, de Fijter JW, Kweekel $\mathrm{DM}$, Swen JJ, et al. Therapeutic drug monitoring of tacrolimus and mycophenolic acid in outpatient renal transplant recipients using a volumetric dried blood spot sampling device. Br J Clin Pharmacol. 2018;84(12):2889-902.

30. Benz-de Bretagne I, Le Guellec C, Halimi JM, Gatault P, Barbet $\mathrm{C}$, Alnajjar A, et al. New sampling strategy using a Bayesian approach to assess iohexol clearance in kidney transplant recipients. Ther Drug Monit. 2012;34(3):289-97.

31. Riff C, Besombes J, Gatault P, Barbet C, Buchler M, Blasco H, et al. Assessment of the glomerular filtration rate (GFR) in kidney transplant recipients using Bayesian estimation of the iohexol clearance. Clin Chem Lab Med. 2020;58(4):577-87.

32. Friedman AN, Strother M, Quinney SK, Hall S, Perkins SM, Brizendine EJ, et al. Measuring the glomerular filtration rate in obese individuals without overt kidney disease. Nephron Clin Pract. 2010;116(3):C224-34.

33. Taubert M, Ebert N, Martus P, van der Giet M, Fuhr U, Schaeffner E. Using a three-compartment model improves the estimation of iohexol clearance to assess glomerular filtration rate. Sci Rep. 2018;8(1):17723.

34. Levey AS, Inker LA. GFR as the "gold standard": estimated, measured, and true. Am J Kidney Dis. 2016;67(1):9-12. 


\section{Authors and Affiliations}

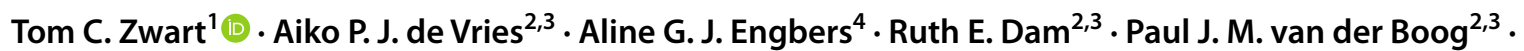

Jesse J. Swen ${ }^{1,5} \cdot$ Ron J. Keizer $^{6}$. R. Neil Dalton ${ }^{7}$ Henk-Jan Guchelaar ${ }^{1,5}$. Johan W. de Fijter ${ }^{2,3}$. Dirk Jan A. R. Moes ${ }^{1,5}$

$\triangle$ Dirk Jan A. R. Moes

D.J.A.R.Moes@lumc.nl

1 Department of Clinical Pharmacy and Toxicology, Leiden University Medical Center, Albinusdreef 2, 2333ZA Leiden, the Netherlands

2 Department of Internal Medicine (Nephrology), Leiden University Medical Center, Leiden, the Netherlands

3 LUMC Transplant Center, Leiden University Medical Center, Leiden, the Netherlands
4 Division of Systems Biomedicine and Pharmacology, Leiden Academic Centre for Drug Research, Leiden University, Leiden, the Netherlands

5 Leiden Network for Personalised Therapeutics, Leiden, the Netherlands

6 InsightRX, San Francisco, CA, USA

7 WellChild Laboratory, Evelina London Children's Hospital, London, UK 